\title{
Immunotherapy for Lewis lung carcinoma utilizing dendritic cells infected with CK19 gene recombinant adenoviral vectors
}

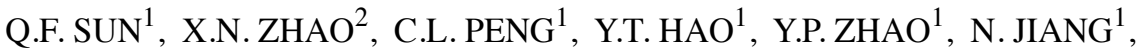 \\ H. XUE ${ }^{1}$, J.Z. GUO ${ }^{1}$, C.H. YUN ${ }^{1}$, B. $\mathrm{CONG}^{1}$ and X.G. ZHAO ${ }^{1}$ \\ ${ }^{1}$ Department of Thoracic Surgery, Second Hospital of Shandong University; ${ }^{2}$ Department of Comprehensive Health II, \\ Provincial Hospital Affiliated to Shandong University, Jinan, Shandong, P.R. China
}

Received June 5, 2015; Accepted August 3, 2015

DOI: $10.3892 /$ or.2015.4231

\begin{abstract}
Dendritic cells (DCs) as 'professional' antigenpresenting cells (APCs) initiate and regulate immune responses to various antigens. DC-based vaccines have become a promising modality in cancer immunotherapy. Cytokeratin 19 (CK19) protein is expressed at high levels in lung cancer and many other tumor cells, suggesting CK19 as a potential tumor-specific target for cancer immune therapy. We constructed a recombinant adenoviral vector containing the CK19 gene (rAd-CK19). DCs transfected with rAd-CK19 were used to vaccinate C57BL/6 mice bearing xenografts derived from Lewis lung carcinoma (LLC) cells. The transfected DCs gave rise to potent CK19-specific cytotoxic T lymphocytes (CTLs) capable of lysing LLC cells. Mice immunized with the rAd-CK19-DCs exhibited significantly attenuated tumor growth (including tumor volume and weight) when compared to the tumor growth of mice immunized with rAd-c DCs or DCs during the 24-day observation period $(\mathrm{P}<0.05)$. The results revealed that the mice vaccinated with the rAd-CK19-DCs exhibited a potent protective and therapeutic antitumor immunity to LLC cells in the subcutaneous model along with an inhibitive effect on tumor growth compared to the mice vaccinated with the rAd-c DCs or DCs alone. The present study proposes a meaningful mode of action utilizing rAd-CK19 DCs in lung cancer immunotherapy.
\end{abstract}

\section{Introduction}

Lung cancer is the leading cause of cancer-related deaths worldwide (1). Despite recent advances in treatment, the

Correspondence to: Dr Xiaogang Zhao, Department of Thoracic Surgery, Second Hospital of Shandong University, 247 Beiyuan Street, Jinan, Shandong 250033, P.R. China

E-mail: xueningent@126.com

Key words: immunotherapy, dendritic cells, cytokeratin 19, Lewis lung carcinoma cells, recombinant adenoviral vectors overall survival of patients with lung cancer remains only $15 \%$ at 5 years, and this declines to less than $2 \%$ in patients with metastatic disease (2). Traditional chemotherapy predominantly kills drug-sensitive cells, leaving behind a heterogeneous population of resistant cells, including those that have the potential to re-populate the tumor or seed new metastatic sites. Immunotherapy has been considered as a valuable treatment for lung carcinoma. It has many advantages including: its specificity to tumor cells and its capability of eliciting a long-lasting immune memory response to kill tumor cells.

Many antitumor vaccines have utilized cell-surface proteins as target molecules, such as livin protein (3-5). Cytokeratin 19 (CK19), a type I cytokeratin, belongs to a group of intermediate filament proteins forming the cytoskeleton. CK19 is the smallest known acidic cytokeratin and is not paired with a basic cytokeratin, unlike the other proteins of this group. In vitro studies of rat lung alveolar epithelial cells have shown high expression of CK19 in intensively proliferating type II pneumocytes (6). Increased expression of this protein was found by immunohistochemical staining in lung cancer cells in humans, and a particularly strong overexpression was observed in cases of squamous cell carcinoma and adenocarcinoma (7). Since CK19 is overexpressed in a variety of human tumors, but poorly or unexpressed in normal differentiated cells, it may be a suitable target for tumor immunotherapy (8). In the present study, we explored an immunization strategy using $\mathrm{H}-2^{\mathrm{b}}$ positive mouse DC2.4s transduced with rAd-CK19 dendritic cells (DCs) to induce the resistance of C57BL/6 mice to Lewis lung carcinoma (LLC) cells. The present study should be valuable for DC-based lung cancer immunotherapy.

\section{Materials and methods}

Mice and cell lines. C57BL/6 mice [SCXK (SU) 2011-0003] (21-35 days of age and 18-22 $\mathrm{g}$ in weight) were purchased from the Cavens Experimental Animal Center (Changzhou, China). Lewis carcinoma cell line (H-2 ${ }^{\text {b }}$ ) was cultivated in RPMI-1640 medium containing antibiotics and $10 \%$ fetal calf serum (BioWit Technologies, Shenzhen, China).

Generally, DCs were extracted from bone marrow (BM) $(9,10)$. Firstly, BM cells were drawn from femurs and tibias of C57BL/6 mice. BM cells were flushed into RPMI-1640 
medium (non-serum) and cultured on plates (6-wells) at $5 \times 10^{6}$ cells/well in normal RPMI-1640 medium, which was cultured with $10 \%$ heat-inactivated fetal bovine serum, murine interleukin (IL)-4 and recombinant murine colony-stimulating factor (GM-CSF). The next day, the non-adherent cells were gently removed, and fresh medium was added with IL-4 and GM-CSF. Half of the culture medium was replaced with fresh medium containing IL- 4 and GM-CSF, every 2 days. Finally, after 7 days, the non-adherent cells were harvested. The present study was approved by the Ethics Committee of the Second Hospital of Shandong University.

Construction of the adenoviral vector encoding CK19. Firstly, we amplified CK19 cDNA from the plasmid pIRES2-EGFPCK19 by polymerase chain reaction (PCR). Then, the products were digested using HindIII and NotI. The shuttle plasmid pDC316-EGFP-cmv (BioWit Technologies) was inserted to construct pDC316-EGFP-cmv-CK19 at the same site, and then it was identified by PCR. HEK-293 cells were transfected with the pDC316-EGFP-cmv-CK19 plasmid. A recombinant adenovirus encoding human CK19 (rAd-CK19) was constructed using AdMax Kit D (BioWit Technologies) (Fig. 1). The transfected cells were harvested among the cells with normal CPE. The cells were repeatedly washed with phosphatebuffered saline (PBS), and the cells were frozen and thawed 3 times at $70^{\circ} \mathrm{C} / 37^{\circ} \mathrm{C}$. The supernatant was collected after the lysate was subjected to centrifugation $(3,000 \mathrm{rpm})$ at $4^{\circ} \mathrm{C}$ for $10 \mathrm{~min}$. This was the preliminary crude lysate containing rAd-CK19, and then the rAd-CK19 went through 3 rounds of amplification in vitro. The titer of the rAd-CK19 passaged was calculated using endpoint dilution assay for 4 times $(11,12)$. The rAd-CK19 was evaluated by the PCR method after being generated.

Assay of the expression of CK19 mRNA in Lewis lung carcinoma (LLC) cells, DCs and CK19-DCs by RT-PCR. The RNA drawn from CK19-DCs was used to evaluated the expression of CK19 by RT-PCR on the basis of the sequences of the multiple clone site of the pcDNA3.1 vector and CK19. The primers for CK19 used for amplification were: upper, 5'-GGT GTCGACCTAGCCAAGAT-3' and lower, 5'-TCTGGATCT GCTCAGAGTGG-3'. The primers for $\beta$-actin were: upper, 5'-TTCTTGGGTATGGAATCCTGTG-3' and lower, 5'-GAG GAGCAATGATCTTGATCTT-3'.

Transduction of DCs with rAd-CK19. The DCs were infected with either rAd-c or rAd-CK19 at a multiplicity of infection (MOI) of 200 and were incubated in medium containing GM-CSF (20 ng/ml) and IL-4 (10 ng/ml) for $2 \mathrm{~h}$. The infected cells were cultured in complete medium for $48 \mathrm{~h}$. Then, the DCs were used for subsequent experiments after being washed with PBS. Cell viability was assessed by trypan blue dye exclusion. We used flow cytometry to evaluate the transduction efficiency of the rAd-CK19-DCs.

Assay of the expression of CK19 in LLC cells, DCs and CK19-DCs by western blotting. rAd-CK19 DCs, LLC cells and rAd-c DCs were used for immunofluorescence technique and western blotting assays. Assays were carried out with an anti-CK19 polyclonal antibody (Shandong Medical College
Jinan, China) via chemiluminscence utilizing an Amersham ECL Advance system with semi-dry transfer.

Analysis using flow cytometry for the phenotype of the DCs. rAd-CK19-DCs, non-infected DCs and rAd-cDCs were harvested and suspended in FACS buffer (PBS with $0.2 \%$ BSA and $0.09 \% \mathrm{NS}$ ) on day 9. LLC cells were immunostained with anti-mouse MHC II, CD80 or CD86 antibodies (PE-labeled) with an isotype-matched control antibody. The antibodies and LLC cells were cultured at $4^{\circ} \mathrm{C}$ for $30 \mathrm{~min}$, and washed with PBS twice. Finally, the DC cells were analyzed using FACScan (BioWit Technologies).

Assay of T cell proliferation. CK19-DCs were treated with mitomycin $\mathrm{C}(25 \mu \mathrm{g} / \mathrm{ml})$ for $30 \mathrm{~min}$ at $37^{\circ} \mathrm{C}$ while the $\mathrm{DC}$ and mock-DC groups were used as controls. After washing with PBS for several times, the cells $\left(4 \times 10^{5}\right.$ cells $\left./ \mathrm{ml}\right)$ were harvested in RPMI-1640 medium (10\% FCS). The cell suspension (100 $\mu 1 /$ well) was added into plates (96-well) which were filled with $\mathrm{CD} 4^{+} \mathrm{T}$ cells $\left(3 \times 10^{5}\right.$ cells/100 $\mu \mathrm{l} /$ well $)$ isolated from the C57BL/6 mice; PBS was used as the control. Finally, the T cell proliferation was determined by MTT assay after 24, 48, 72 and $96 \mathrm{~h}$.

Tumor immunization and prophylactic therapy. To evaluate the immunotherapeutic effect of the rAd-CK19-DCs, we used C57BL/6 mice (21-35 days of age; the mice were randomly divided into 3 groups, 6 mice/group) which were injected with tumor cells (LLC cells at $5 \times 10^{5} /$ mouse) into the right flanks. When the subcutaneous tumors grew to $4-6 \mathrm{~mm}$, the mice were treated by subcutaneously injection with rAd-CK19-DCs, rAd-cDCs or DCs $\left(5 \times 10^{5} /\right.$ mouse) into the left flanks every 3 days, for a total of 3 times. Approximately 14 days after the last injection, the mice were sacrificed (cervical dislocation), and this was recorded as the date of death. To evaluate the antitumor effect, the tumor was measured with digital calipers every 2 days. Tumor volumes were calculated using the formula: $\mathrm{D} \mathrm{x} \mathrm{d} \mathrm{d}^{2} \times 0.52$ ( $\mathrm{D}=$ length, $\mathrm{d}=$ width). After sacrifice of the mice, the tumors were removed and weighed $(\mathrm{g})$.

Cytotoxic T lymphocyte (CTL) assay. In order to evaluate the ability of rAd-CK19-DCs to induce tumor-specific CTLs, C57BL/6 mice were subcutaneously injected with DCs, rAd-CK19-DCs or rAd-c DCs for 2 times every 7 days. Splenocytes were collected and cultured in complete medium after the final injection. Non-adherent cells were harvested and cultured in plates (24-well) with mitomycin-C-treated LLCs $\left(10^{6}\right.$ cells $\left./ \mathrm{ml}\right)$. Then the splenocytes were cultured in medium containing $10 \mathrm{U} / \mathrm{ml}$ of IL-2 at $37^{\circ} \mathrm{C}$ for 5 days. The effector cells (E) treated with LLCs, and target cells (T) were put into 96-well plates at different E:T ratios (60:1, 40:1 and 20:1). The plates were centrifuged at $250 \mathrm{x}$ g for $4 \mathrm{~min}$, and then the cells were collected. The negative controls were the cells without effector cells. The cytotoxicity ratio was calculated using the following formula: Experimental release - spontaneous release/maximum release - spontaneous release (\%).

Statistical analysis. All statistical analyses were performed using the SPSS 13.0 software package. The Student's t-test and $\chi^{2}$ test were used to compare data from two groups. Differences 
A AdMax $^{\mathrm{TM}}$ for generation of adenovirus vectors

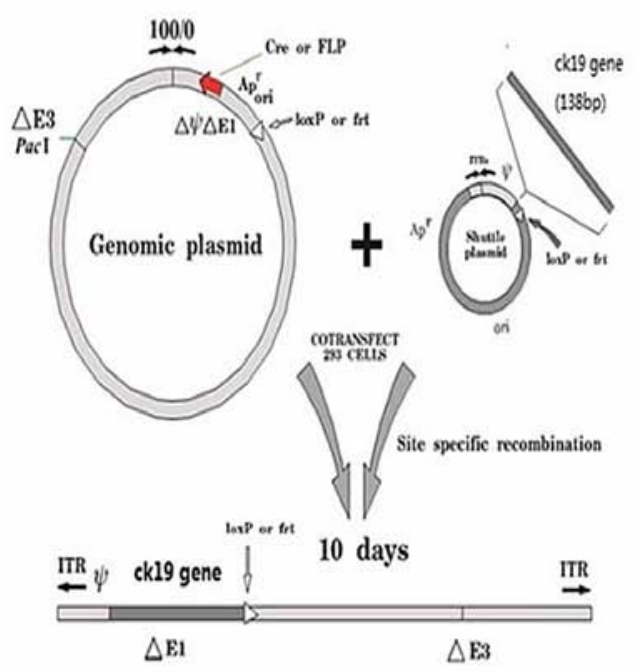

B
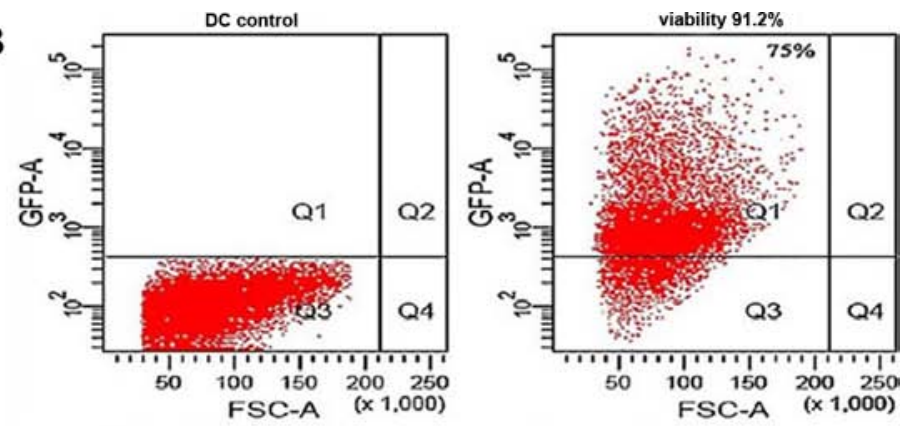

C

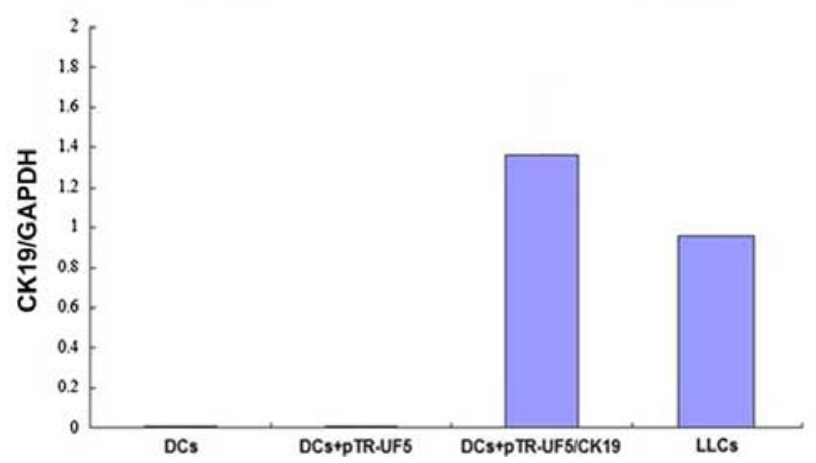

Figure 1. Construction of rAd-CK19 and transduction of DCs with rAd-CK19 and cell viability. (A) Demonstrative figure of the construction of the recombinant adenovirus and CK19 gene, using AdMax system. The shuttle plasmid pDC316-EGFP-cmv-CK19 was transfected into HEK-293 cells with the plasmid pBHGlox E1, 3 Cre containing 5 type adenovirus genome. Then the recombinant, replication-deficient adenovirus rAd-CK19 gene was generated. (B) Transduction of DCs with rAd-CK19 and cell viability. After infection for $48 \mathrm{~h}$, GFP expression was evaluated and the percentage of viable cells was evaluated using trypan blue staining; Q1, \% of GFP-positive cells by flow cytometry. (C) Assay of CK19 mRNA expression in LLC cells, rAd-CK19-DCs and DCs by RT-PCR.

A

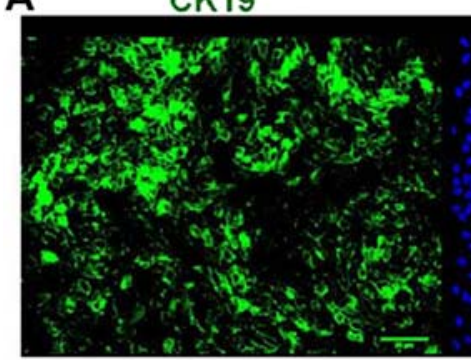

DAPI CK19+ DAPI

B

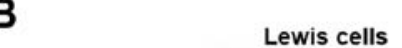

DC2.4 cells

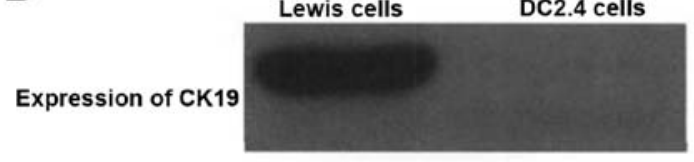

C
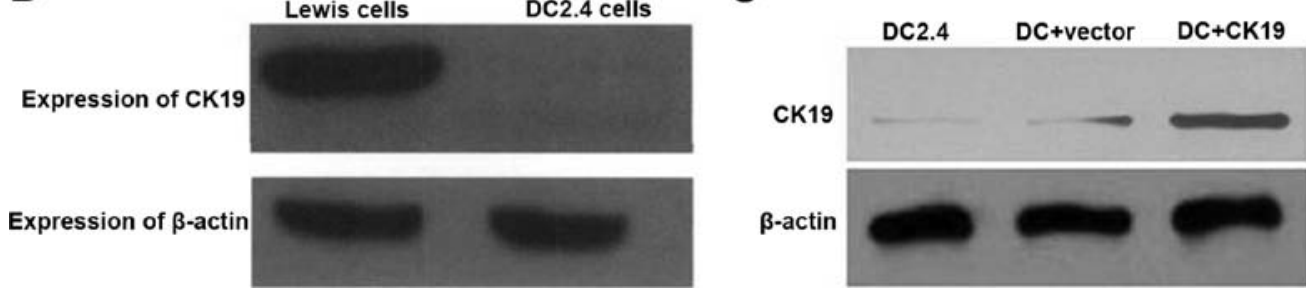

Figure 2. (A) Identification of CK19 expression in LLC cells by immunofluorescence (magnification, x100). (B) Expression of CK19 protein in LLC cells and DC2.4s by western blotting. (C) CK19 expression in DC2.4 and rAd-CK19-DCs. DC+vector was used as a negative control.

associated with a P-value of 0.05 were considered statistically significant.

\section{Results}

Preparation of the rAd-CK19. Following successful construction of the plasmid pDC316-EGFP-cmv-CK19 which was identified by PCR, HEK-293 cells were transfected with this plasmid as shown in Fig. 1A. HEK-293 cells were infected with rAd-CK19, and 3 days later the expression of GFP was also monitored by flow cytometry (Fig. 1B).

Transduction of the DCs with the adenoviral vector encoding CK19. DCs transfected with rAd-CK19 were analyzed for transduction efficiency after 7 days. The results of flow cytometry showed that $\sim 80 \%$ of DCs were positive for GFP. When the MOI of gene transduction was 200 , its cell viability was $>80 \%$ (Fig. 1B). 

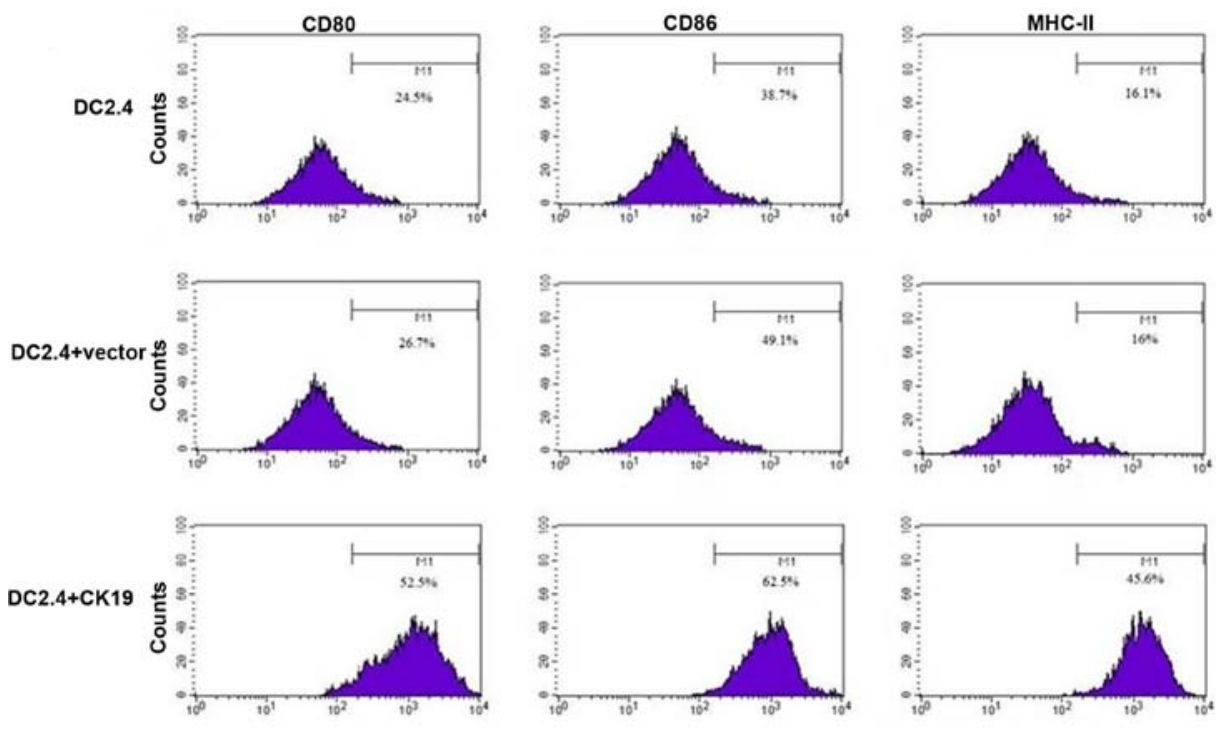

JDC2.4

DC+vector

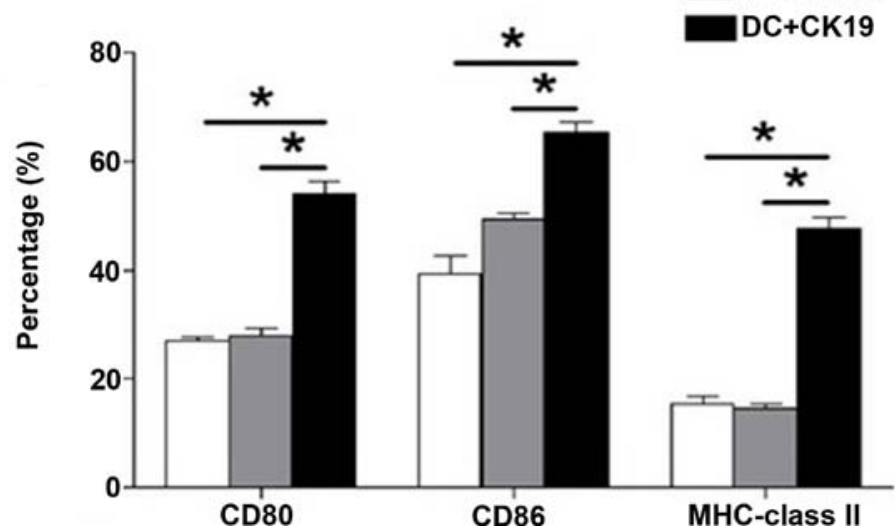

Figure 3. Variation of surface markers on CK19-DCs. The expression levels of MHC class II, CD86 or CD80 on CK19-DCs, DCs or vector-DCs were analyzed by FCM, and CK19-DCs exhibited markedly increased expression of MHC class II, CD80 and CD86.

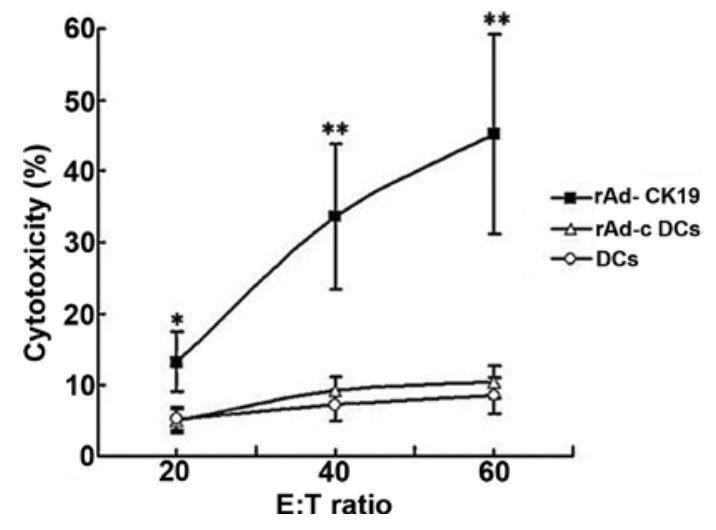

Figure 4. Induction of specific T cells by mice vaccinated with rAd-c DCs. Mice were subcutaneously vaccinated 3 times during a 3-day interval with DCs (o), rAd-CK19-DCs (匹) or rAd-c DCs $(\triangle)$. After the last vaccination, spleen cells were isolated and stimulated with mitomycin C-treated LLC cells for 5 days. The stimulated cells were then assayed for cytolytic function using LLC cells as target cells. Results are shown as means $\pm \mathrm{SD},{ }^{*} \mathrm{P}<0.01 \mathrm{com}-$ pared with rAd-c DCs or DCs; ${ }^{* *} \mathrm{P}<0.001$ compared with rAd-c DCs or DCs.

Expression of CK19 in the rAd-CK19 DCs and LLC cells. As shown in Figs. 1 and 2, CK19 mRNA and protein were monitored in LLC cells. CK19 mRNA and protein were also detected in the rAd-CK19-DCs, while poor expression was observed in the DCs and rAd-c DCs.

Effect on the DCs after infection with rAd-CK19. To assess the effect on DC activity after infection with rAd-CK19, we analyzed three phenotypes of the rAd-c DCs, DCs or rAdCK19-DCs by flow cytometry. The results showed (Fig. 3) that expression levels of MHC class II, CD80 and CD86 on rAd-CK19-DCs were significantly higher than levels on the rAd-c-DCs, which proved that rAd-infected DCs had enhanced MHC class II, CD80 and CD86 expression levels compared to the expression levels in the normal DCs. Thus, we concluded that infection with the recombinant adenovirus led to the maturation of the DCs. In contrast, there was no apparent effect on DC differentiation and activation by CK19 gene infection.

Activity of tumor-specific CTLs. As shown in Fig. 4, the E cells vaccinated with rAd-CK19-DCs induced significantly killing responses by CTLs against lung cancer cells. On the contrary, the control groups vaccinated with DCs or rAd-c DCs expressed less lysis. The results proved that E cells vaccinated with the rAd-CK19-DCs induced tumor-specific CTL responses. 


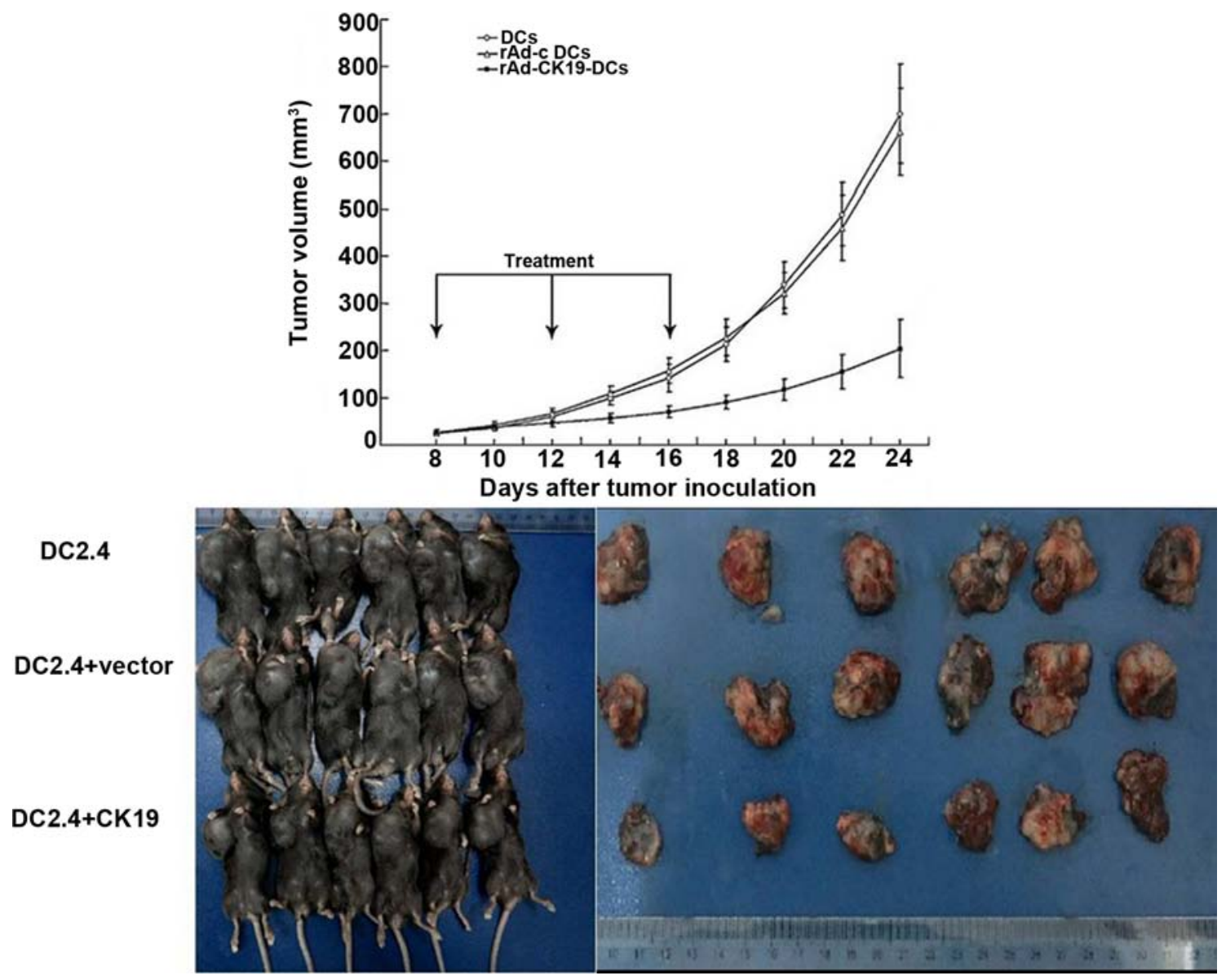

Figure 5. Effect of rAd-CK19-DCs on tumor growth in mice. LLC cells were subcutaneously injected into mice into the right flanks on day 1 . When tumors grew to 4-6 mm, the mice were divided into groups with similar tumor size (6 mice/group). Then the mice were injected with rAd-c DCs ( $\triangle$ ), rAd-CK19 DCs (匹) or DCs (o). Tumor volume was measured every 2 days. Mean \pm SD of tumor volume is shown. The CK19-DC vaccine significantly attenuated the tumor growth as compared to that in the control groups.

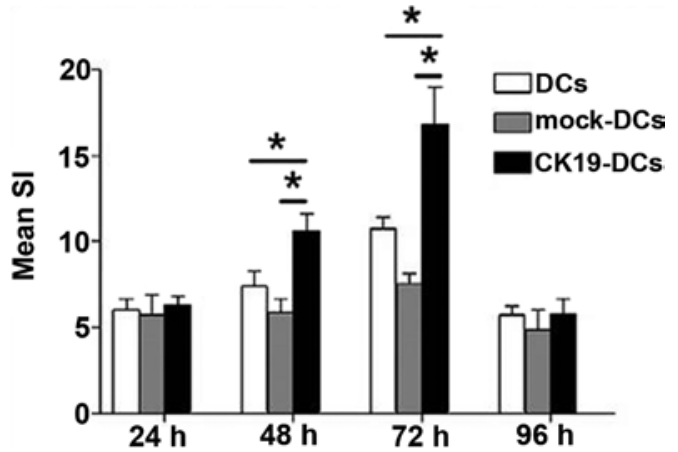

Figure 6. Capacity of CK19-DCs to sitimulate T cell proliferation. Proliferation of T cells was evaluated by MTT assay. The SI yielded 10.30 \pm 0.93 and $15.78 \pm 1.95$ in the CK19-DCs compared with $5.67 \pm 0.58$ and $6.99 \pm 1.51$ in the rAd-c DCs $(\mathrm{P}<0.01)$, and $7.12 \pm 0.66$ and $9.63 \pm 0.89$ in the DCs at 24 and $48 \mathrm{~h}$. The results are presented as the mean $\pm \mathrm{SD}(\mathrm{n}=5),{ }^{*} \mathrm{P}<0.01$.

Antitumor effects of immunization with rAd-CK19 DCs. We used a mouse LLC cell tumor model to evaluate the immunotherapeutic effect of rAd-CK19-DCs. Mice vaccinated with rAd-CK19-DCs exhibited markedly induced antitumor effects compared to the control group during the 24-day observation period. As shown in Fig. 5, immunization with rAd-CK19-DCs significantly attenuated the growth of tumors as compared to the tumor growth in the control groups immunized with rAd-c
DCs or DCs $(\mathrm{P}<0.01)$. Tumor volume in the control groups was $\sim 1$-fold larger than that in the treated groups. There was no difference in tumor volume between the two control groups $(P>0.05)$. The results of the tumor weights were the same as the tumor sizes. The average weights of the tumors (immunized with rAd-c DCs and DCs) were 2.800 and $2.788 \mathrm{~g}$, while the average weight of the tumors (immunized with rAd-CK19 DCs) was $1.035(\mathrm{P}<0.01)$. These results suggested that the rAd-CK19-DC vaccine evoked a cytotoxic antitumor immune response to the target cells.

Proliferation of $T$ cells driven by rAd-CK19 DCs. CK19-DCs had a higher capability to evoke special $\mathrm{T}$ cell proliferation than that noted in the control groups (rAdc-DCs and DCs). The proliferation of T cells, stimulated with CK19-DCs began at $48 \mathrm{~h}$ as compared with $\mathrm{T}$ cell proliferation of the control group which reached the highest peak after $72 \mathrm{~h}$. The study suggested that CK19-DCs have the capability to stimulate $\mathrm{T}$ cell proliferation (Fig. 6).

\section{Discussion}

Host antigen-presenting cells (APCs) are critical for the presentation of tumor antigens (13). DCs are the most effective APCs, which have the capability to stimulate special immune 
response dependent on the T cells. Antigen-loaded DCs migrate to lymphoid tissues and relate to naive $\mathrm{T}$ cells, where the labeled DCs stimulate immune responses dependent on co-stimulatory and adhesive molecules on the cell surface (9).

The ability to induce original $\mathrm{CD} 8^{+} \mathrm{T}$ and $\mathrm{CD} 4^{+}$cells, and to expand those cells at sites of tumor antigen expression provides an efficient method for priming $\mathrm{T}$ cells. Injecting DC vaccines to secondary lymphoid organs is a critical step to stimulate the initiation of a primary immune response, where DCs encounter naive $T$ cells. Almost at the same time, the $\mathrm{CD}^{+} \mathrm{T}$ and $\mathrm{CD}^{+} \mathrm{T}$ cells infiltrate into the tumor site (14).

The use of a DC-based tumor vaccine is important for advanced stage tumors with poor responsiveness to chemotherapy, such as lung cancer. DCs can contain different proteins or peptides of tumors. As the source of antigens for immunotherapy, the lysate of tumor cells offers potential target antigens, which should induce a greater T-cell response against multiple known or unknown tumor-associated antigens of individual cancer cells (15). The immunotherapy of DCs loaded with tumor antigens has been proven to be an effective antitumor strategy in preclinical tumor therapy and some have been used in clinical therapy research, such as in melanoma and pancreatic cancer therapy (16-19).

Vaccination of DCs infected with adenovirus vectors encoding special or broad tumor antigens can stimulate protective and antigen-specific CTL responses and immunity to kill target cells (20-22). Transduction with replicationdefective and recombinant adenovirus vectors encoding target genes is an efficient method for gene transfer into DCs $(23,24)$. Xie et al and Zhou et al have shown that DCs pulsed with tumor antigens livin- $\alpha$ and XAGE- $1 \mathrm{~b}$ induced significant immune responses by CTLs against LLC cells $(25,26)$.

In the present study, we generated rAd-CK19-DCs which expressed the CK19 gene at high levels through adenovirus transduction at an MOI of 200 (Fig. 1), and transduction efficiency of cell viability was $>80 \%$ (Fig. 1). In addition, maturation, enhancement and activation of $\mathrm{T}$ cell stimulatory capacity have also been achieved for DC adenovirus-mediated transduction $(27,28)$.

According to previous studies $(12,29)$, adenovirus-mediated transduction induced maturation and upregulated surface antigen expression of DCs through a nuclear B-dependent mechanism. On the other hand, adenovirus vectors infected with target tumor antigens delivered immunogenic adenovirus genes and proteins, which induced special cellular responses (30). In the present study, the viral vector had no significant effect on the expression of the DC phenotype at an MOI of 200 and the upregulation of MHC class II, CD80 and CD86 by adenoviral vector was also detected in the rAdcDCs (Fig. 3).

Cytokeratins, which belong to a family of 20 individual polypeptides, form an intracellular network of filaments that participates in maintaining the structural integrity of the cell. The expression pattern of cytokeratin polypeptides varies with the cell type and changes during transformation of normal epithelial cells into malignant cells in many types of cancer (31). Moreover, cytokeratins were applied in evaluation of the degree to which a tissue is abnormally differentiated (32). CK19 is the smallest member of the cytokeratin family, and its expression is highly tissue-specific. Many studies have shown that CK19 expression is correlated with LNM in various solid malignancies (33-36), including the liver, colon, stomach, pancreas, biliary tract and breast $(37,38)$.

CK19, a type I cytokeratin, belongs to a group of intermediate filament proteins forming the cytoskeleton. CK19 is the smallest known acidic cytokeratin and is not paired with a basic cytokeratin, unlike the other proteins of this group. In vitro studies of rat lung alveolar epithelial cells have shown high expression of CK19 in intensively proliferating type II pneumocytes (6). Meanwhile, CK19 mRNA was assessed to investigate the prognostic significance of circulating cancer cells in peripheral blood of patients with non-small cell lung cancer (36).

Increased expression of this protein was found using immunohistochemical methods in lung cancer, particularly overexpression in squamous cell carcinoma and adenocarcinoma (7). Thus, according to these findings, we used an immunization strategy using the CK19 gene as the target. The immune system is tolerant to many self antigens, which is a potential obstacle for any immunotherapeutic strategy that relies on a vaccination against specific antigens. The tumor vaccination protocol with the addition of cytokines or other immune modulators may help to halt the immune escape of tumor cells. Steitz et al (39) reported that, in C57BL/6 mice, immunization with recombinant adenovirus vector encoding human xenogeneic tyrosinase-related protein 2 induced protection against the growth of B16 melanoma cells. Another study showed that, in syngeneic Fischer rats, a human xenogeneic DNA vaccine stopped the anti-apoptotic protein survivin to inhibit the growth of F98 glioblastoma (40). In the same way, the homologous epitopes encoded by the xenogeneic DNA act as molecular mimics recognized as nonself, resulting in an immune response against host self gene products. Downregulating specific tumor antigens may help in preventing tumor escape.

In conclusion, in the present study, we used the human xenogeneic CK19 antigen as a target to stop the immune tolerance of mice to the CK19 antigen. According to our results (Fig. 4), the mice vaccinated with the rAd-CK19 DCs generated special CTLs, which could recognize CK19-derived antigens stimulating significant killing responses. To test the antitumor effect of the rAd-CK19 DC vaccine in vivo, we used C57BL/6 mice with LLC as a tumor model with which to evaluate the antitumor effect of the rAd-CK19-DC vaccine. Vaccination with rAd-CK19 DCs prevented the growth of the LLC tumors in the mice. Thus, we considered that it provided significant protection against tumor growth (Fig. 5). In contrast, the control groups vaccinated with DCs or rAd-c DCs did not exhibit effective tumor protection. According to the above, we conclude that rAd-CK19-DC vaccine could generate an effective specific CK19 antitumor effect. In summary, rAdCK19-DC-based vaccine may offer a promising approach to cancer immunotherapy in clinical trials, and may play an important role in fighting lung cancer.

\section{Acknowledgements}

The present study was supported by the Shandong Provincial Natural Science Foundation, China (nos. ZR2009CM108 and ZR2013HQ061). 


\section{References}

1. Ferlay J, Shin HR, Bray F, Forman D, Mathers C and Parkin DM Estimates of worldwide burden of cancer in 2008: GLOBOCAN 2008. Int J Cancer 127: 2893-2917, 2010.

2. Collins LG, Haines C, Perkel R and Enck RE: Lung cancer: Diagnosis and management. Am Fam Physician 75: 56-63, 2007.

3. Moscatello DK, Ramirez G and Wong AJ: A naturally occurring mutant human epidermal growth factor receptor as a target for peptide vaccine immunotherapy of tumors. Cancer Res 57 1419-1424, 1997

4. Heimberger AB, Crotty LE, Archer GE, et al: Epidermal growth factor receptor VIII peptide vaccination is efficacious against established intracerebral tumors. Clin Cancer Res 9: 4247-4254, 2003.

5. Ciesielski MJ, Kazim AL, Barth RF and Fenstermaker RA: Cellular antitumor immune response to a branched lysine multiple antigenic peptide containing epitopes of a common tumor-specific antigen in a rat glioma model. Cancer Immunol Immunother 54: 107-119, 2005.

6. Paine R, Gaposchkin D, Kelly C and Wilcoxen SE: Regulation of cytokeratin expression in rat lung alveolar epithelial cells in vitro. Am J Physiol 269: L536-L544, 1995.

7. Kosacka M and Jankowska R: The prognostic value of cytokeratin 19 expression in non-small cell lung cancer. Pneumonol Alergol Pol 75: 317-323, 2007 (In Polish).

8. Overwijk WW, Tsung A, Irvine KR, et al: gp100/pmel 17 is a murine tumor rejection antigen: Induction of 'self'-reactive, tumoricidal T cells using high-affinity, altered peptide ligand. J Exp Med 188: 277-286, 1998

9. Banchereau J and Steinman RM: Dendritic cells and the control of immunity. Nature 392: 245-252, 1998.

10. Steinman RM and Dhodapkar M: Active immunization against cancer with dendritic cells: The near future. Int J Cancer 94 459-473, 2001

11. Nyberg-Hoffman C, Shabram P, Li W, Giroux D and AguilarCordova E: Sensitivity and reproducibility in adenoviral infectious titer determination. Nat Med 3: 808-811, 1997.

12. Morelli AE, Larregina AT, Ganster RW, et al: Recombinant adenovirus induces maturation of dendritic cells via an NF-kappaB-dependent pathway. J Virol 74: 9617-9628, 2000

13. Huang AY, Golumbek P, Ahmadzadeh M, et al: Role of bone marrow-derived cells in presenting MHC class I-restricted tumor antigens. Science 264: 961-965, 1994

14. Sharma S, Stolina M, Luo J, et al: Secondary lymphoid tissue chemokine mediates $\mathrm{T}$ cell-dependent antitumor responses in vivo. J Immunol 164: 4558-4563, 2000.

15. Fields RC, Shimizu K and Mulé JJ: Murine dendritic cells pulsed with whole tumor lysates mediate potent antitumor immune responses in vitro and in vivo. Proc Natl Acad Sci USA 95: 9482-9487, 1998.

16. Schnurr M, Galambos P, Scholz C, et al: Tumor cell lysate-pulsed human dendritic cells induce a T-cell response against pancreatic carcinoma cells: An in vitro model for the assessment of tumor vaccines. Cancer Res 61: 6445-6450, 2001.

17. Mitchell MS, Harel W, Kan-Mitchell J, et al: Active specific immunotherapy of melanoma with allogeneic cell lysates Rationale, results, and possible mechanisms of action. Ann NY Acad Sci 690: 153-166, 1993

18. Kirk CJ and Mulé JJ: Gene-modified dendritic cells for use in tumor vaccines. Hum Gene Ther 11: 797-806, 2000.

19. Klein C, Bueler H and Mulligan RC: Comparative analysis of genetically modified dendritic cells and tumor cells as therapeutic cancer vaccines. J Exp Med 191: 1699-1708, 2000.

20. Kim S, Lee JB, Lee GK and Chang J: Vaccination with recombinant adenoviruses and dendritic cells expressing prostate-specific antigens is effective in eliciting CTL and suppresses tumor growth in the experimental prostate cancer. Prostate 69: 938-948, 2009.

21. Song W, Kong HL, Carpenter H, Torii H, Granstein R, Rafii S, Moore MA and Crystal RG: Dendritic cells genetically modified with an adenovirus vector encoding the cDNA for a model antigen induce protective and therapeutic antitumor immunity. $J$ Exp Med 186: 1247-1256, 1997.
22. Ribas A, Butterfield LH, Hu B, et al: Generation of T-cell immunity to a murine melanoma using MART-1-engineered dendritic cells. J Immunother 23: 59-66, 2000.

23. Kaplan JM, Yu Q, Piraino ST, et al: Induction of antitumor immunity with dendritic cells transduced with adenovirus vectorencoding endogenous tumor-associated antigens. J Immunol 163 : 699-707, 1999.

24. Cho HI, Kim HJ, Oh ST and Kim TG: In vitro induction of carcinoembryonic antigen (CEA)-specific cytotoxic $\mathrm{T}$ lymphocytes by dendritic cells transduced with recombinant adenoviruses. Vaccine 22: 224-236, 2003.

25. Zhou Q, Guo AL, Xu CR, An SJ, Wang Z, Yang SQ and Wu YL: A dendritic cell-based tumour vaccine for lung cancer: Full-length XAGE-1b protein-pulsed dendritic cells induce specific cytotoxic T lymphocytes in vitro. Clin Exp Immunol 153: 392-400, 2008.

26. Xie J, Xiong L, Tao X, Li X, Su Y, Hou X and Shi H: Antitumor effects of murine bone marrow-derived dendritic cells infected with xenogeneic livin alpha recombinant adenoviral vectors against Lewis lung carcinoma. Lung Cancer 68: 338-345, 2010.

27. Rea D, Schagen FH, Hoeben RC, et al: Adenoviruses activate human dendritic cells without polarization toward a T-helper type 1-inducing subset. J Virol 73: 10245-10253, 1999.

28. Jonuleit H, Tüting T, Steitz J, et al: Efficient transduction of mature $\mathrm{CD} 83^{+}$dendritic cells using recombinant adenovirus suppressed T cell stimulatory capacity. Gene Ther 7: 249-254, 2000.

29. Miller G, Lahrs S, Shah AB and DeMatteo RP: Optimization of dendritic cell maturation and gene transfer by recombinant adenovirus. Cancer Immunol Immunother 52: 347-358, 2003.

30. Yang Y, Li Q, Ertl HC and Wilson JM: Cellular and humoral immune responses to viral antigens create barriers to lungdirected gene therapy with recombinant adenoviruses. J Virol 69: 2004-2015, 1995

31. Zhong LP, Zhao SF, Chen GF, Ping FY, Xu ZF and Hu JA: Increased levels of CK19 mRNA in oral squamous cell carcinoma tissue detected by relative quantification with real-time polymerase chain reaction. Arch Oral Biol 51: 1112-1119, 2006.

32. Galus R and Wodarski K: Importance of the cytokeratins in cancer diagnosis. Pol Merk Lek 135: 209-211, 2007.

33. Xenidis N, Ignatiadis M, Apostolaki S, et al: Cytokeratin-19 mRNA-positive circulating tumor cells after adjuvant chemotherapy in patients with early breast cancer. J Clin Oncol 27: 2177-2184, 2009

34. Van Trappen PO, Gyselman VG, Lowe DG, et al: Molecular quantification and mapping of lymph-node micrometastases in cervical cancer. Lancet 357: 15-20, 2001.

35. Visser M, Jiwa M, Horstman A, et al: Intra-operative rapid diagnostic method based on CK19 mRNA expression for the detection of lymph node metastases in breast cancer. Int $\mathrm{J}$ Cancer 122: 2562-2567, 2008.

36. Chen TF, Jiang GL, Fu XL, Wang LJ, Qian H, Wu KL and Zhao S: CK19 mRNA expression measured by reverse-transcription polymerase chain reaction (RT-PCR) in the peripheral blood of patients with non-small cell lung cancer treated by chemoradiation: An independent prognostic factor. Lung Cancer 56: 105-114, 2007.

37. van Sprundel RG, van den Ingh TS, Desmet VJ, et al: Keratin 19 marks poor differentiation and a more aggressive behaviour in canine and human hepatocellular tumours. Comp Hepatol 9: 4, 2010.

38. Ohshio G, Imamura T, Okada N, Yamaki K, Suwa H, Imamura M and Sakahara H: Cytokeratin 19 fragment in serum and tissues of patients with pancreatic diseases. Int J Pancreatol 21: 235-241, 1997.

39. Steitz J, Brück J, Steinbrink K, Enk A, Knop J and Tüting T: Genetic immunization of mice with human tyrosinase-related protein 2: Implications for the immunotherapy of melanoma. Int J Cancer 86: 89-94, 2000.

40. Fenstermaker RA and Ciesielski MJ: Immunotherapeutic strategies for malignant glioma. Cancer Control 11: 181-191, 2004. 